\title{
Photovoltaic Temperature Challenges and Bismuthene Monolayer Properties
}

\author{
Farzaneh Ghasemzadeh*, Mahdi Esmaeilzadeh**, Mostafa Esmaeili Shayan**** \\ * Ph.D. The student in Condensed Matter Physics, Iran University of Science and Technology, Tehran, Iran \\ **Department of Physics, Professor of Physics, Iran University of Science and Technology, Tehran, Iran \\ ***** Ph.D. The student in Renewable Energy Engineering, Tarbiat Modares University, Tehran, Iran \\ (FGH.7192@yahoo.com, Mahdi@iust.ac.ir, Mostafa.Esmaeili@modares.ac.ir) \\ ॠCorresponding Author; Mostafa Esmaeili Shayan, 3355133116, Tel: +982133562109, \\ Fax: +982133562109, Mostafa.esmaeili@modares.ac.ir
}

Received: 14.11.2020 Accepted:20.12.2020

\begin{abstract}
International Energy Agency addresses critical challenges for the study, development, and implementation of renewable energy technologies for the long term. In the energy market, the biggest problem is to maximize production and reduce losses. In the present analysis, the usable density approach used a linear potential system amplified plane waves to conduct various monolayer Bismuthenes, including structural, optical, and electronic properties, under different biaxial stresses. The findings showed that the single-layer Bismuthene free train has 0,599 eV (9,597-20 J) extreme ribbon energy and can be used as one of the 2D material layers for developing cheap solar cells. This substance also has strong absorption and low light loss and $8 \%$ metallic properties. It is also necessary to use this substance as a covering of the upper layers of solar heat receptors.
\end{abstract}

Keywords Renewable Energy, Efficiency, Electronic Properties, Optical Properties, Monolayer Bismuthene, Photovoltaic.

\section{Introduction}

Two-dimensional (2D) materials have significant applications in transistors. Some materials have special applications due to their unique structures. One-dimensional structures such as 2-dimensional material graphene [1], onedimensional and two-dimensional German [2], onedimensional and two-dimensional silicon[3], and twodimensional statin [4] are used to evaluate applications have become. The results show that this type of material's zero bandgaps has limited their use to transistor materials and field effect (FET) applications. Recently, a new family of 2D materials including III-nitride monolayers $\mathrm{XN}(\mathrm{X}=\mathrm{B}, \mathrm{Al}, \mathrm{Ga}$ and In) [5], phosphorene [6], $\mathrm{MoS}_{2}$, antimonide [7], and bismuthene [8-9] are known as a wide range bandgap from (0$4.58 \mathrm{eV})$ which is very suitable for modern application and FETs.

Among these 2D atomic layer materials, monolayer bismuthene with a buckled 2D structure show promising properties like or such as (1) high carrier mobility from 300 (ML) to $5.7 \times 10^{6} \mathrm{~cm}^{2} / \mathrm{V} . \mathrm{s}$ (in bulk) [10], (2) stability at room temperature [11], (3) low thermal [12]. Kistanov et al. [13] have studied the oxidation mechanisms and suggest maintaining their structural integrity under environmental conditions. In the other study, Bhuvaneswari, Nagarajan, and Chandiramouli [14] have investigated the adsorption of bismuthene nanosheets as an efficient nerve agent sensor by using SIESTA code in 2019. Also, Guo et al. [15] have been studied the interfacial properties between bismuthene and metal (Al, Ag, Au, Ir, Ti, and Pt) contacts by using firstprinciple energy ban calculation and quantum transport simulation. They guide bismuth monolayers and metals' surface properties for special applications in the metal industry and electrical systems. In 2018, Chen et al. [16] reported on the optical properties of monolayer bismuthene in electric fields. They show that the threshold frequency of $2 \mathrm{D}$ bismuthene becomes zero under the critical electric field.

Due to the unique properties of monolayer bismuth and the existence of density performance theory, it was decided to investigate the electronic and optical properties of bismuth monolayer under tensile and compressive stresses. In 2020, the studies have revealed that the single-layer phases of bismuth are stable at a high temperature, which is an excellent property to use in photovoltaic systems [17].

The purpose of this research includes the technical calculation of bismuthene with select properties and technical study of electrical, optical, and intrinsic properties for use in 
energy systems. The results of the analysis of this material are affected by stress analysis in the two-dimensional state.

\section{Materials and Methods}

Using the full potential and functional theory framework, the density of different properties of monolayer bismuth was implemented and coded. The WIEN-2K commercial package has been used for this purpose. Simultaneously, the main references are the basis for decision-making to continue research activities [18]. The generalized gradient approximation medium (GGA) and the Perdew-BurkeErnzrhoff medium are used for correlation exchange [19]. The $14 \times 14 \times 1$ and $27 \times 27 \times 1 \mathrm{k}$-grids in the first Brillouin zone are used for electronic and optical calculations, respectively. The convergence parameter $\mathrm{RK}_{\max }$ was set to 7 , which $\mathrm{R}$ stands for the smallest muffin-tin radius, $K_{\max }$ is the maximum size of reciprocal lattice vectors. The Fourier expanded charge density was truncated at $\mathrm{G}_{\max }=14.0 \mathrm{Bohr}^{-1}$. A $20 \AA$ vacuum space was constructed to avoid the periodical computations, we have distinguished the core electrons of $\mathrm{Bi}$ $\left(1 s^{2} 2 s^{2} 2 p^{6} 3 s^{2} 3 p^{6} 3 d^{10} 4 s^{2} 4 p^{6} 4 d^{10} 5 s^{2}\right)$ and the valence electrons of $\mathrm{Bi}\left(5 p^{6} 4 f^{14} 5 d^{10} 6 s^{2} 6 p^{3}\right)$.

\section{Result and Discussion}

\subsection{Structural and Electronic properties}

We have optimized the buckled two dimensional (2D) bismuthene with a lattice constant of $4.34^{\circ} \mathrm{A}$, which is in good agreement with previous work(s) [20]. Figure 1 shows the scientific view of monolayer bismuth in different directions.

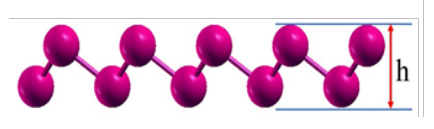

(a) Lateral view

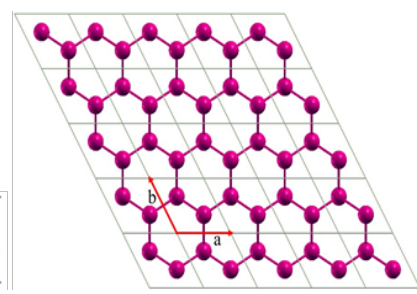

(b) top view
Fig. 1. Stable and entire structure of bismuth monolayer: (a) Lateral view (h is buckling factor) and (b) View from the top (axes a and b, respectively: zigzag direction).

Tensile and compressive stresses were used in the system with constant grid scaling. Equation (1) shows the tensile and compressive stress calculator.

$\eta=\frac{a_{0}-a}{a_{0}} \times 100$

Where $a_{0}$ is the optimized lattice constant of bismuthene (strain-free lattice constant), and $a$ is the lattice constant for nanosheet under strain. The positive and negative values of $\eta$ refer to compressive and tensile strain, respectively. The amount of strain ranges from $\eta=-8 \%$ (tensile) to $\eta=8 \%$ (compressive) by steps of $4 \%$. Our results, such as lattice constant, bond length, buckling parameters, and bond angle for strain-free and strained monolayer bismuthene, are summarized in Table 1.
Table 1. Calculated values for lattice constant $(a)$, bond length $(l)$, buckling parameter $(h)$, and bond angle $(\theta)$ of monolayer bismuthene under different magnitudes of compressive and tensile strain.

\begin{tabular}{ccccc}
\hline$\eta$ & $a(\AA)$ & $l(\AA)$ & $h(\AA)$ & $\theta$ (degree) \\
\hline$+8 \%$ & 3.993 & 2.983 & 1.892 & 84.03 \\
$+4 \%$ & 4.166 & 3.001 & 1.804 & 87.71 \\
0 & 4.340 & 3.043 & 1.730 & 90.96 \\
$-4 \%$ & 4.514 & 3.090 & 1.661 & 93.83 \\
$-8 \%$ & 4.687 & 3.141 & 1.597 & 96.51 \\
\hline
\end{tabular}

The calculated values of these parameters after structural optimization are found to be $4.340,3.043,1.730 \AA$, and $90.96^{\circ}$, which corresponds to the lattice constant, bond length, buckling parameter, and bond angle, respectively, and are in good agreement with previous studies. It was evident that by applying strain from $\eta=-8 \%$ to $\eta=8 \%$, the lattice Constant bond length and bond angle are increased while the buckling parameter is decreased compared to the strain-free case. To confirm a response of the bandgap of the monolayer $\mathrm{Bi}$ to the different kinds of strain, the band structures of bismuthene along the high-symmetry $\Gamma-\mathrm{K}-\mathrm{M}-\Gamma$ path and total density of states (TDOS) for strain-free, compressive, and tensile strain cases are presented in Fig. 2.

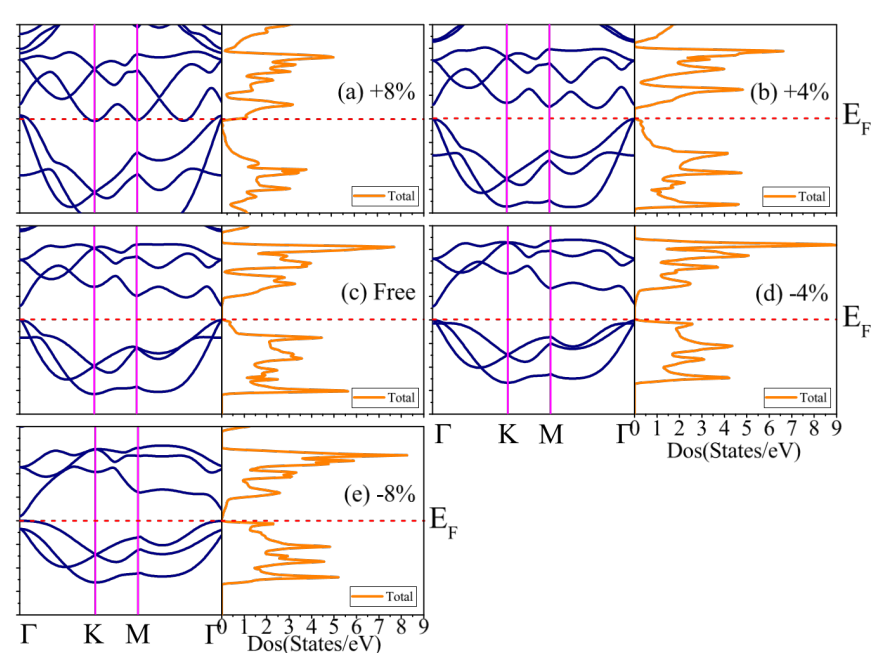

Fig. 2. Electronic band structure and corresponding total density of states of strained 2D-Bi for (a) $\eta=+8 \%$, (b) $\eta=$ $+4 \%$, (c) $\eta=0$, (d) $\eta=-4 \%$, (e) $\eta=-8 \%$.

The band structure of 2D bismuthene at the strain-free condition demonstrates a direct bandgap of $0.599 \mathrm{eV}$ with both VBM (valence band maximum) and CBM (conduction band minimum) located at $\Gamma$, shown in Fig. 2 c. Fig. 2 shows that monolayer bismuthene is compressed $(\eta>0)$ when the CBM lies on the $\mathrm{M}-\Gamma$ path, so a direct to indirect bandgap transition occurs. The bandgap's magnitude decreases to $0.470 \mathrm{eV}$ when the compressive strain reaches $\eta=+8 \%$ the monolayer $\mathrm{Bi}$ turns into a metal. Since recognizing the critical point of transition from semiconductor to metal is essential, we have calculated the energy bandgap ranging from $\eta=+4 \%$ to $\eta=$ $+8 \%$ with step 1 . The results show at $\eta=+7 \%$, a transition 
from semiconductor to metal occurs. On the other hand, by exerting tensile strain to the system $(\eta<0)$, the bandgap energy is reduced from $0.599 \mathrm{eV}$ (at $\eta=0$ ) to $0.196 \mathrm{eV}$ (at $\eta=$ $-8 \%$ ) but is still direct with both the CBM and VBM located at $\Gamma$ point. We have also plotted the variation of the electronic bandgap of monolayer bismuthene as a function of strain. The critical point that semiconductor-metal transition happens is marked with a star symbol in Fig. 3.

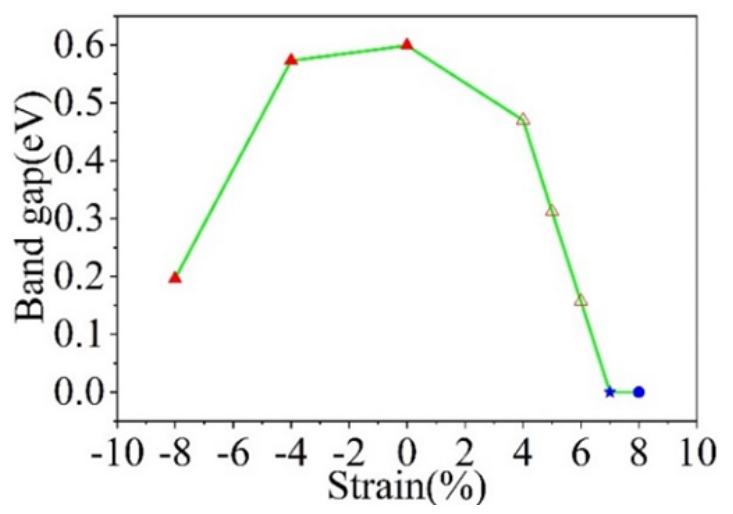

Fig. 3. Energy band gap versus strain for 2D-Bi under compressive and tensile strain. Filled and unfilled symbols represent the direct and indirect bandgap, respectively. The star symbol stands for the critical point of the semiconductor to metal transition.

It was evident that by extending and compressing the system, the energy bafnd gap is decreased, and at $\eta=+7 \%$, it shows metal properties. Table 2 has been listed the exact values of calculated electronic band gap values and directions of $2 \mathrm{D}$ bismuthene under compressive and tensile strain.

Table 2. Calculated electronic band gap values $(\mathrm{eV})$ and its directions of monolayer bismuthene under different magnitudes of compressive and tensile strain.

\begin{tabular}{llllll}
\hline$\eta$ & $-8 \%$ & $-4 \%$ & 0 & $+4 \%$ & $+8 \%$ \\
\hline$E_{\mathrm{g}-\mathrm{Bi}}(\mathrm{eV})$ & 0.196 & 0.573 & 0.599 & 0.47 & - \\
& $\Gamma \rightarrow \Gamma$ & $\Gamma \rightarrow \Gamma$ & $\Gamma \rightarrow \Gamma$ & $\mathrm{K} \rightarrow \Gamma$ & - \\
\hline
\end{tabular}

The partial density of states of monolayer bismuthene under compressive and tensile strain to specify the contribution of orbitals in the structure of bandgap energy, as illustrated in Fig. 4. Also, states analysis's partial density for strain-free monolayer bismuthene shows that the VBM has primarily derived from $p_{x+y}$ states, while the CBM is mainly dominated by $p_{z}$ state. At $\eta=+4 \%$, which occurs directly to indirect bandgap transition, the CBM mainly consists of porbitals and s-orbital of $\mathrm{Bi}$. The DOS has zero value at $\eta=$ $+8 \%$ indicating the semiconductor to metal transition. Under tensile strain, the contribution of orbitals is the same as an unstrained case.

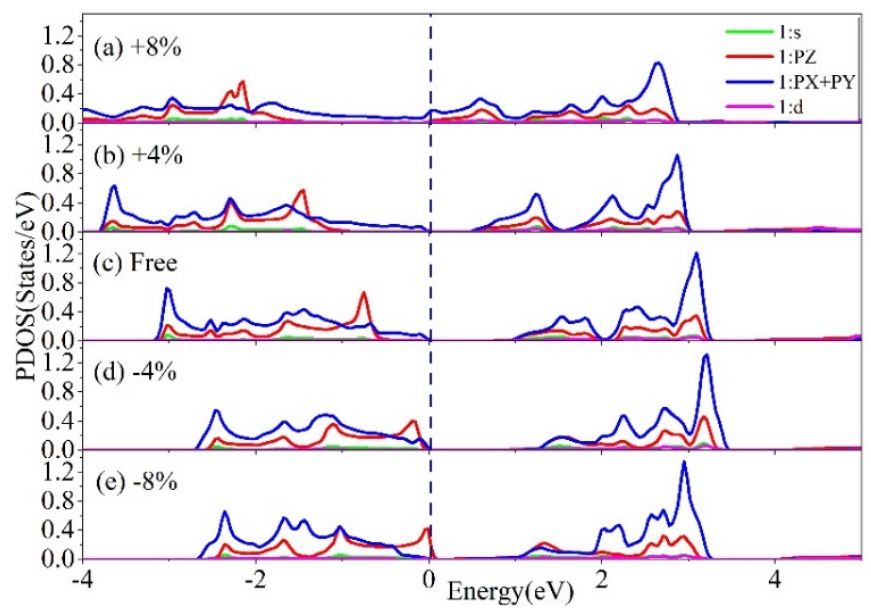

Fig. 4. The partial density of states of $2 \mathrm{D}-\mathrm{Bi}$ for (a) $\eta=+8 \%$, (b) $\eta=+4 \%$, (c) $\eta=0$, (d) $\eta=-4 \%$, (e) $\eta=-8 \%$.

\subsection{Optical properties}

Since the electronic calculations indicated the bandgap of monolayer bismuthene could be tuned by applying compressive and tensile strains, the study of the optical properties of bismuthene under strain is vital to practical applications of nanotechnology and optoelectronics. In the study [21] using stochastic fuzzy random phase approximation (RPA) method as a reference, monolayer bismuth's optical properties, including real and imaginary part of the dielectric function, refractive index, reflectance, and band transfer properties under different stresses, have been investigated. Is. Equation (2) describes the optical response of the electromagnetic field complex dielectric $\varepsilon(\omega)$ in different energy bands, and the continuum is used.

$\varepsilon(\omega)=\varepsilon_{1}(\omega)+i \varepsilon_{2}(\omega)$

Equation (2) seeks to receive the optical response of the system. In this regard, $\epsilon 1(\omega)$ and $\epsilon 2(\omega)$ are the real and imaginary parts of the performance of complex optical systems. In the source [22], the section of the imagination of dielectric performance $\epsilon 2(\omega)$ is related to the transmission between bands. This variable is used in the occupied ( $\mathrm{kn})$ and unoccupied $\left(\mathrm{kn}^{\prime}\right)$ state in the relation.

$\varepsilon_{2}(\omega)=\frac{v e^{2}}{2 \pi \hbar m^{2} \omega^{2}} \int \mathrm{d} \mathbf{k} \sum_{k k^{\prime}}\left[\left|\left\langle k n|\mathbf{P}| k n^{\prime}\right\rangle\right|^{2} \times f(k n)\left(1-f\left(k n^{\prime}\right)\right) \delta\left(E_{n k}-E_{n^{\prime} k}-\hbar \omega\right)\right]$

Where $\hbar \omega$ is the energy of the incident photon, $\mathbf{P}$ is the momentum operator, $|k n\rangle$ is the eigenfunction with eigenvalue $E_{n k}$, and $f(k n)$ is a Fermi distribution function. The relation [23] is the same as the Kramers-Kroning relation, and P represents this part's original value. This relation represents the real part of the dielectric function $\epsilon 1(\omega)$.

$\varepsilon_{1}(\omega)=1+\frac{2}{\pi} P \int_{0}^{\infty} \frac{\varepsilon_{2}\left(\omega^{\prime}\right) \omega^{\prime} \mathrm{d} \omega^{\prime}}{\omega^{\prime 2}-\omega^{2}}$

Real and imaginary parts of bismuth dielectric performance under different strain values for both parallels and perpendicular polarization of the electric field ( $E_{\|}$and $\left.E_{\perp}\right)$ are depicted in Fig. 5. 

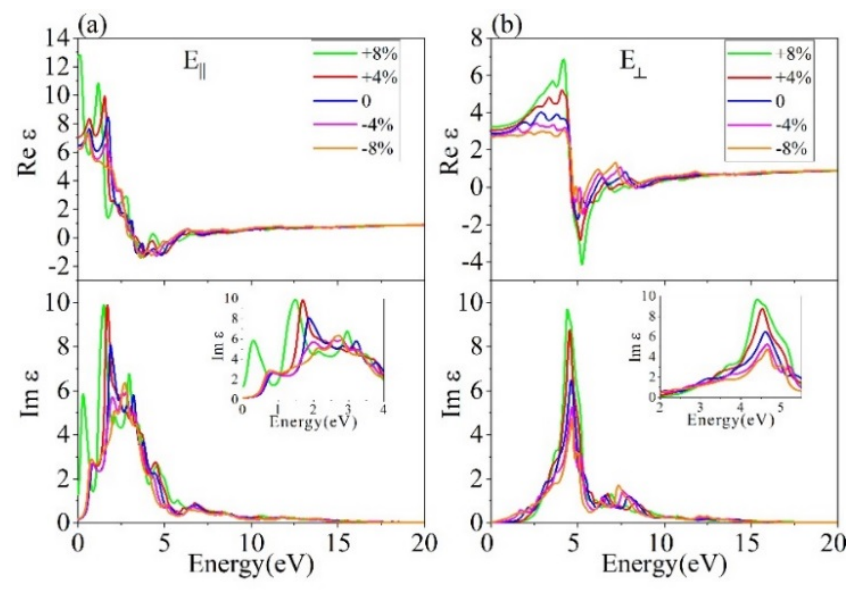

Fig. 5. Two-dimensional dielectric performance diagram of bismuthene under parallel tensile stress (a) and real perpendicular polar stress (b).

If Equation (4) were used, then the static values of the actual parts of the monolayer bismuth for both poles and the function Di $(\epsilon 1(0))$ for the different stress values are reported in Table 3.

Table 3. Calculated static dielectric constant $\epsilon_{1}(0)$ of $2 \mathrm{D}-\mathrm{Bi}$ under compressive and tensile for parallel and perpendicular polarizations.

\begin{tabular}{llllll}
\hline$\eta$ & $-8 \%$ & $-4 \%$ & 0 & $+4 \%$ & $+8 \%$ \\
\hline$E_{\|}$ & 6.21 & 6.19 & 6.68 & 7.06 & 12.68 \\
$E_{\perp}$ & 2.70 & 2.75 & 2.87 & 3.06 & 3.23 \\
\hline
\end{tabular}

Its value for the unstrained case is 6.68 and 2.87 for parallel and perpendicular polarization. By increasing compressive strain from $\eta=0$ to $\eta=+8 \%$, the static dielectric constant for both polarizations increases, while it is decreased by increasing tensile strain from $\eta=0$ to $\eta=-8 \%$ in both directions, as shown in Fig. 5. We have also calculated the interband transitions for strain-free monolayer bismuthene, which correspond to these prominent peaks. From Fig. 5 it can be seen that there is one central peak for parallel and two prominent peaks for perpendicular polarization. The probable interband transition is listed in Table. 4, and the corresponding energy levels are determined in Fig. 6.

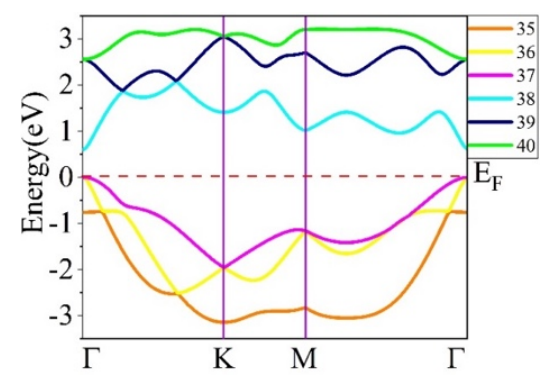

Fig. 6. Energy band structure of strain-free 2D-Bi. The determined numbers were used to investigate the probable optical transitions.

By applying compressive strain $(\eta>0)$, the imaginary part of the dielectric function of monolayer bismuthene shows the shift in peaks towards the lower energy region, were causes redshift, while these peaks shift to the higher energy by applying tensile strain $(\eta<0)$ which blue-shift occurs (Fig. 5).

Table 4. Calculated optical interband transitions of strain-free 2D-Bi for both polarizations.

\begin{tabular}{lllll}
\hline$p_{1}(\|)$ & $36 \rightarrow 38$ & $37 \rightarrow 38$ & & \\
\hline$\left.p_{2}(\|)\right)$ & $35 \rightarrow 39$ & $35 \rightarrow 40$ & $37 \rightarrow 38$ & $37 \rightarrow 39$ \\
$p(\perp)$ & $35 \rightarrow 38$ & $36 \rightarrow 39$ & $36 \rightarrow 40$ & $37 \rightarrow 40$ \\
\hline
\end{tabular}

The reflectivity spectra of monolayer bismuthene for both parallel and perpendicular polarization are shown in Fig. 7. One can be found the reflectivity at low energy for parallel polarization is large. Therefore, the value of the transition is low. In perpendicular polarization, reflectivity in the approximate range of $3.8 \mathrm{eV}$ to $6.5 \mathrm{eV}$ is large.

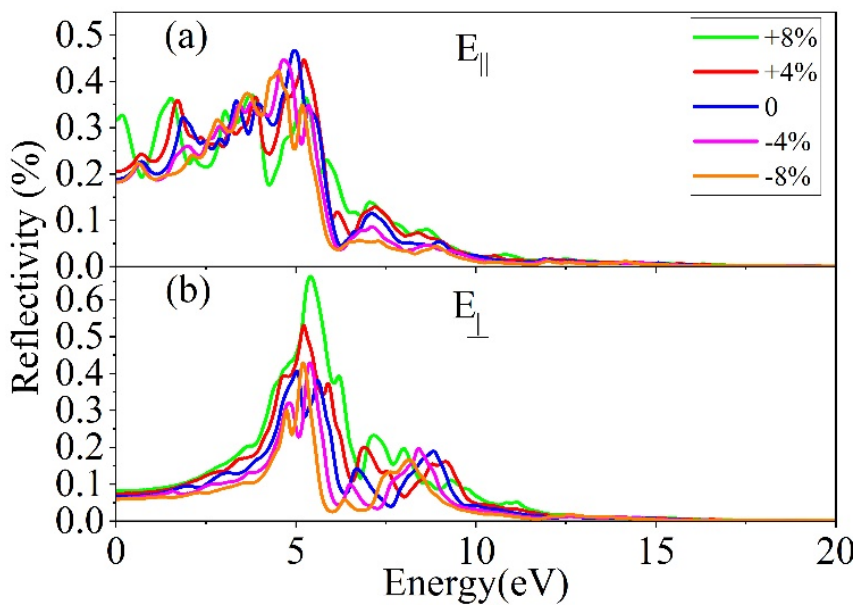

Fig. 7. The reflectivity spectra of $2 \mathrm{D}-\mathrm{Bi}$ under compressive and tensile (a) parallel and (b) perpendicular polarizations.

Consequently, the transition in perpendicular polarization at low energy is more extensive than its value in parallel. As a result, the refractive index of bismuth monolayer and 2D-Bi in compressive stress values in parallel and vertical dipoles is calculated. The static bismuth refractive index of $2 \mathrm{D}-\mathrm{Bi}(\mathrm{n}(0))$ in the no-load state is $2.54 \mathrm{eV}$ and $1.69 \mathrm{eV}$ for parallel and vertical poles, respectively. The exact values of the refractive index for a different amount of strains are recorded in Table.5.

Table 5. The refractive index of two-dimensional bismuthene 2D-Bi n (0) under different parallel and vertical polar stress values.

\begin{tabular}{llllll}
\hline$\eta$ & $-8 \%$ & $-4 \%$ & 0 & $+4 \%$ & $+8 \%$ \\
\hline$n \|$ & 2.49 & 2.49 & 2.54 & 2.66 & 3.56 \\
$n_{\perp}$ & 1.64 & 1.66 & 1.69 & 1.75 & 1.80 \\
\hline
\end{tabular}

In two-dimensional bismuth, as shown in Figure 8, in the range of $-8 \%$ to $+8 \%$, the static refractive index for both polarizations increases with increasing stress. The static refractive index in parallel polarized direction occurs at 0.12 , $1.92,0.58 \mathrm{eV}$ for $+8 \%, 0,-8 \%$, respectively. These values in 
perpendicular polarized direction are $4.26,4.45,4.34 \mathrm{eV}$ for the same value of strains, respectively.

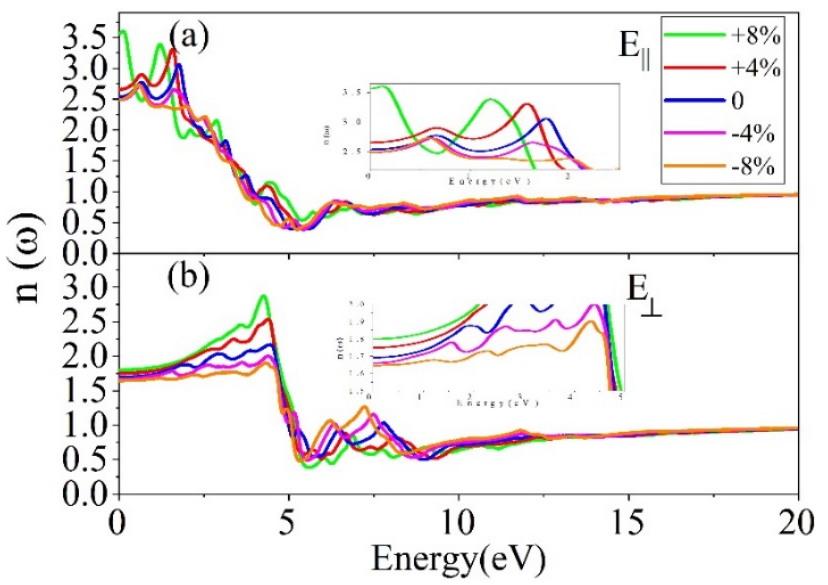

Fig. 8. Refractive index of 2D-Bi under compressive and tensile for (a) parallel and (b) perpendicular polarizations.

\section{Conclusion}

The electrical, structural, and optical properties of twodimensional bismuth monolayer material under the influence of various compressive and tensile stresses have been investigated for the proposed material for use in the photovoltaic industry. These stresses affect the material's bond structure properties and energy. Monolayer bismuthene freestrain has a direct $0.599 \mathrm{eV}$ bandgap with CBM and VBM at a high-symmetry point. By applying compressive strain, the monolayer bismuthene undergoes a direct to indirect bandgap transition (almost everywhere to $\mathrm{M}$ ), while remaining unchanged under tensile strain. When a homogeneous biaxial strain was applied, the bandgap could be tuned from $0.196 \mathrm{eV}$ to $0.47 \mathrm{eV}$ for $-8 \%<=+8 \%$. By analyzing the partial density of the state diagrams, the bismuthene nanosheet acquires metallic properties under $+8 \%$ stress. By calculating the dielectric function, the static dielectric constant at all stresses was accurately obtained, i.e. 6,68 and 2,87 for stress-free conditions for parallel and perpendicular polarizations. Since the extreme peaks in the diagrams of the imaginary part of the dielectric function represent the transitions of the optical interband, we measured these transitions at low energy levels and determined exactly which transition corresponds to each peak. Also by calculating the reflectivity spectrum, we found that the most reflectivity exists for parallel polarization at energies below $6 \mathrm{eV}$, so the transition in this energy range is minimal. The most perpendicular polarization reflectivity spectrum occurs at approximately $5 \mathrm{eV}$. The results of the calculation of the bismuthene monolayer refractive index show an increase in the static refractive index from $-8 \%$ to $+8 \%$ for both polarizations, the lowest value for parallel (perpendicular) polarization is 2.49 (1.64) and the maximum value for parallel (perpendicular) polarization is 3.56 (1.80) and this spectrum has reached a constant value after an e. The studied static refraction index, interband optical transitions, and reflectivity spectra under compressed and tensile biaxial strain show that the transitions of the interband can be calculated with a band structure. The 2D bismuthene adsorbs light in the infrared regimes and adsorption spreads across a spectral spectrum as a broadband optical response. Because of its large-scale optical response, bismuthene is commonly used in solar cells, light emission units, photovoltaic systems, and photodetectors.

\section{References}

[1] A.K. Geim, K.S. Novoselov, The rise of graphene, Nat. Mater. 6 (2007) 183-191. https://doi.org/10.1038/nmat1849.

[2] W. Shi, W. Gu, X. Ma, Electronic structure of twodimensional germanene crystals, Emerg. Mater. Res. 5 (2016) 114-118. https://doi.org/10.1680/jemmr.15.00027.

[3] M. Shakeri, Mobin Ghasemzadeh, Farzaneh Afkani, F. Kanjouri, Electronic and Optical Properties of Penta-BP5 Monolayer. In: 7th Int. Congr. Nanosci. Nanotechnol., Tehran, 2018.

[4] G.R. Bhimanapati, N.R. Glavin, J.A. Robinson, 2D Boron Nitride: Synthesis and Applications, in: Semicond. Semimetals, Academic Press Inc., 2016: pp. 101-147. https://doi.org/10.1016/bs.semsem.2016.04.004.

[5] F. Ghasemzadeh, F. Kanjouri, Strain effect on the electronic properties of III-nitride nanosheets: Ab-initio study, Sci. China Technol. Sci. 61 (2018) 535-541. https://doi.org/https://doi.org/10.1007/s11431-017-9177-1.

[6] J. Yan, G. Luo, B. Xiao, H. Wu, Z. He, Y. Cao, Origin of high fill factor in polymer solar cells from semiconducting polymer with moderate charge carrier mobility, Org. Electron. 24 (2015) $125-130$. https://doi.org/10.1016/J.ORGEL.2015.05.034.

[7] Mostafa Esmaeili Shayan, Solar Energy, and Nuclear Power. Economic Requirements and Hindrances, GRIN Verlag, 2020. https://books.google.com/books?id=KkL8DwAAQBAJ.

[8] M.S. Esmaeili, G. Najafi, Energy-Economic Optimization of Thin Layer Photovoltaic on Domes and Cylindrical Towers, Int. J. Smart Grid - ijSmartGrid. 3 (2019) 84-91. https://www.ijsmartgrid.ijrer.org/index.php/ijsmartgridnew/a rticle/view/61 (accessed July 8, 2019).

[9] M. Azadbakht, E. Esmaeilzadeh, M. Esmaeili-Shayan, Energy consumption during impact cutting of canola stalk as a function of moisture content and cutting height, J. Saudi Soc. $\begin{array}{lllll}\text { Agric. } & \text { Sci. } & 14 & \text { (2015) } & \text { 147-152. }\end{array}$ https://doi.org/10.1016/j.jssas.2013.10.002.

[10] I. V. Provornaya, I. V. Filimonova, L. V. Eder, V.Y. Nemov, E.A. Zemnukhova, Formation of energy policy in Europe, taking into account trends in the global market, in Energy Reports, Elsevier Ltd, 2020: pp. 599-603. https://doi.org/10.1016/j.egyr.2019.09.032.

[11] P. Ares, F. Aguilar-Galindo, D. Rodríguez-San-Miguel, D.A. Aldave, S. Díaz-Tendero, M. Alcamí, F. Martín, J. Gómez-Herrero, F. Zamora, Mechanical Isolation of Highly Stable Antimonene under Ambient Conditions, Adv. Mater. 28 (2016) 6332-6336. https://doi.org/10.1002/adma.201602128. 
[12] M. Esmaeili Shayan, S. Esmaeili Shayan, Factors Impacting Customer Relationship Performance in Social CRM Case Study: Saipa Automotive Company, 2020. https://www.grin.com/document/942698 (accessed November 6, 2020).

[13] A.A. Kistanov, S.K. Khadiullin, K. Zhou, S. V. Dmitriev, E.A. Korznikova, Environmental stability of bismuthene: Oxidation mechanism and structural stability of 2D pnictogens, J. Mater. Chem. C. 7 (2019) 9195-9202. https://doi.org/10.1039/c9tc03219c.

[14] R. Bhuvaneswari, V. Nagarajan, R. Chandiramouli, Electronic properties of novel bismuthene nanosheets with adsorption studies of G-series nerve agent molecules - a DFT outlook, Phys. Lett. Sect. A Gen. At. Solid State Phys. 383 (2019). https://doi.org/10.1016/j.physleta.2019.125975.

[15] Y. Guo, F. Pan, M. Ye, X. Sun, Y. Wang, J. Li, X. Zhang, H. Zhang, Y. Pan, Z. Song, J. Yang, J. Lu, Monolayer Bismuthene-Metal Contacts: A Theoretical Study, ACS Appl. Mater. Interfaces. 9 (2017) 23128-23140. https://doi.org/10.1021/acsami.7b03833.

[16] R.-B. Chen, D.-J. Jang, M.-C. Lin, M.-F. Lin, Optical properties of monolayer bismuthene in electric fields, Opt. Lett. 43 (2018) 6089. https://doi.org/10.1364/ol.43.006089.

[17] W.C. Liu, L. Xu, M.-C. Lin, T.-C. Leung, H.-Y. Hsu, Optical Properties of Monolayer Bismuthene under Electric
Field based on First-principles Calculations, Bull. Am. Phys. Soc. Volume 65, Number 1 (2020).

[18] M. Azadbakht, M.E. Shayan, H. Jafari, Investigation of Long Shaft Failure in John Deere 955 Grain Combine Harvester under Static Load, Univers. J. Agric. Res. 1 (2013) 70-73. https://doi.org/10.13189/UJAR.2013.010305.

[19] M. Azadbakht, H. Jafari, M. Esmaeili Shayan, The Surveying of Exerted Stresses on Gearbox Gears in MF285 Tractor, Univers. J. Agric. Res. 06 (2013) 129-133. https://doi.org/10.13189/UJAR.2013.010402.

[20] F.F. Yun, D.L. Cortie, X.L. Wang, Tuning the electronic structure in stanene/graphene bilayers using strain and gas adsorption, Phys. Chem. Chem. Phys. 19 (2017) 25574 25581. https://doi.org/10.1039/c7cp03678g.

[21] X. Ren, P. Rinke, C. Joas, M. Scheffler, Random-phase approximation and its applications in computational chemistry and materials science, n.d.

[22] E.J.C. Dias, F.J. García De Abajo, Fundamental Limits to the Coupling between Light and 2D Polaritons by Small Scatterers, ACS Nano. 13 (2019) 5184-5197. https://doi.org/10.1021/acsnano.8b09283.

[23] Causality and the Kramers-Kronig relations, (n.d.). http://lampx.tugraz.at/ hadley/ss2/linearresponse/causality.p hp (accessed November 6, 2020). 